\title{
On Boolean algebras of conditionals and their logical counterpart
}

\author{
Tommaso Flaminio ${ }^{1}$, Lluis Godo ${ }^{2}$, and Hykel Hosni ${ }^{3}$ \\ ${ }^{1}$ Dipartimento di Scienze Teoriche e Applicate, Università dell'Insubria \\ Via Mazzini 5, 21100 Varese, Italy. \\ Email: tommaso.flaminio@uninsubria.it \\ ${ }^{2}$ Artificial Intelligence Research Institute (IIIA - CSIC) \\ Campus de la Univ. Autònoma de Barcelona s/n, 08193 Bellaterra, Spain. \\ Email: godo@iiia.csic.es \\ 3 Department of Philosophy, University of Milan, \\ Via Festa del Perdono 7 - 20122 Milano, Italy \\ Email: hykel.hosni@unimi.it
}

\begin{abstract}
This paper sheds a novel light on the longstanding problem of investigating the logic of conditional events. Building on the framework of Boolean algebras of conditionals previously introduced by the authors, we make two main new contributions. First, we fully characterise the atomic structure of these algebras of conditionals. Second, we introduce the logic of Boolean conditionals (LBC) and prove its completeness with respect to the natural semantics induced by the structural properties of the atoms in a conditional algebra as described in the first part. In addition we outline the close connection of LBC with preferential consequence relations, arguably one of the most appreciated systems of non-monotonic reasoning.
\end{abstract}

Keywords. Conditionals Events; Uncertain Reasoning; Boolean algebra of Conditionals; Non-monotonic reasoning.

\section{Introduction}

Conditionals play a fundamental role both in qualitative and in quantitative uncertain reasoning, see e.g. $[15,7,1,14,9,12,6]$. In the former, conditionals constitute the core focus of non-monotonic reasoning $[10,8,11]$. In quantitative uncertain reasoning, conditionals are central both for conditional probability, and more generally, for conditional uncertainty measures [5].

This paper builds on [4], where a Boolean algebra structure for conditionals was proposed with the goal of clarifying the relationship between conditional probabilities and simple probabilities on conditional events. The approach of considering (measure-free) conditionals as Boolean objects departs from previous ones in the literature where conditionals are mainly considered as three-valued objects, proposing different definitions for the operations between consitionals, see e.g. $[2,3,7,9,13]$. For a comparison, the reader may consult [4, Sect. 3.1]. 
Intuitively, a Boolean algebra of conditional events, a BAC algebra for short, is an algebra built up over a Boolean algebra of plain events $A=(A, \wedge, \vee, \neg, \top, \perp)$ in which we allow basic conditionals, i.e. syntactic objects of the form $(a \mid b)$ with $a \in A$ and $b \in A^{\prime}=A \backslash\{\perp\}$, to be freely combined with the usual Boolean operations, subject to the following plausible constraints (recall that in any Boolean algebra $A$, if $a, b \in A$, then $a \leq b$ iff $a \wedge b=a)$ :

- the conditional $(T \mid T)$ will be the top element of the algebra, while $(\perp \mid T)$ will be the bottom;

- given $b \in A^{\prime}$, we want the set of conditionals $\{(a \mid b): a \in A\}$ to be the domain of a Boolean subalgebra, and in particular when $b=T$, then we want this subalgebra to be isomorphic to $A$;

- in a conditional $(a \mid b)$ we can equivalently replace the consequent $a$ by $a \wedge b$, that is, we require the conditionals $(a \mid b)$ and $(a \wedge b \mid b)$ to be equivalent;

- if $a \leq b \leq c$ then the result of conjunctively combining the conditionals $(a \mid b)$ and $(b \mid c)$ yields the conditional $(a \mid c)$.

This last condition captures a form of restricted transitivity and is clearly inspired by the chain rule of conditional probabilities: $P(a \mid b) \cdot P(b \mid c)=P(a \mid c)$ whenever $a \leq b \leq c$.

The purpose of this paper is to put BAC algebras on firm logical footing. To this end, we make two main new contributions. First, we fully characterise the atomic structure of BAC algebras, a problem that was left open in [4]. This is done in Section 3. Second, in Section 4, we introduce the logic of Boolean conditionals (LBC) and prove its completeness with respect to the natural semantics induced by the structural properties of the atoms in a conditional algebra. In Section 5 we conclude with a result to the effect that LBC is indeed a preferential consequence relation, in the sense of the well-known System P, see e.g. [10,11].

\section{Boolean algebras of conditionals}

Boolean algebras of conditionals, introduced and investigated in [4], are built as follows. Let $A=(A, \wedge, \vee, \neg, \top, \perp)$ be a Boolean algebra and let $A^{\prime}=A \backslash\{\perp\}$.

The construction starts with by considering the Boolean algebra of terms freely generated by the pairs $(a, b) \in A \times A^{\prime}$, that will be denoted

$$
\mathcal{F}\left(A \times A^{\prime}\right)=\left(\mathcal{F}\left(A \times A^{\prime}\right), \wedge^{*}, \vee^{*}, \neg^{*}, \top^{*}, \perp^{*}\right) .
$$

Notice that all pairs $(a, b) \in A \times A^{\prime}$ are such that $b>\perp$. This is motivated by the fact that we are avoiding, in this paper, to consider counterfactual conditionals. Since we want conditionals to satisfy a number of properties, what we do is to consider the greatest subalgebra of $\mathcal{F}\left(A \times A^{\prime}\right)$ where these properties hold. Technically speaking, we define it as a quotient algebra by a suitable congruence relation. Namely, consider the following elements in $\mathcal{F}\left(A \times A^{\prime}\right)$, where we use $\delta\left(c, c^{\prime}\right)$ to denote the element $c \leftrightarrow^{*} c^{\prime}$ (of $\left.\mathcal{F}\left(A \times A^{\prime}\right)\right)$ for any $c, c^{\prime} \in \mathcal{F}\left(A \times A^{\prime}\right)$ :

(t1) $\delta\left((y, y), \top^{*}\right)$ for every $y \in A^{\prime}$, 
(t2) $\delta\left((x, y) \wedge^{*}(z, y),(x \wedge z, y)\right)$ for every $x, z \in A$ and $y \in A^{\prime}$,

(t) $\delta(\neg *(x, y),(\neg x, y))$ for every $x \in A, y \in A^{\prime}$,

(t4) $\delta((x \wedge y, y),(x, y))$ for every $x \in A, y \in A^{\prime}$,

$(\mathbf{t} 5) \delta\left((x, z),(x, y) \wedge^{*}(y, z)\right)$ for every $x \in A$ and $y, z \in A^{\prime}$ such that $x \leq y \leq z$.

Let $\mathfrak{C}$ be the filter of $\mathcal{F}\left(A \times A^{\prime}\right)$ generated by the set of all the instances of the above terms (t1-t5). We hence define the congruence relation $\equiv_{\mathfrak{C}}$ in the following way: $c \equiv_{\mathfrak{C}} c^{\prime}$ if $\delta\left(c, c^{\prime}\right) \in \mathfrak{C}$.

Definition 1. For every Boolean algebra A, we say that the quotient algebra $\mathcal{C}(A)=\mathcal{F}\left(A \times A^{\prime}\right) / \equiv_{\mathfrak{C}}$ is the Boolean algebra of conditionals of $A$, the BAC algebra of $A$ for short.

Since $\mathcal{C}(A)$ is a quotient of a free Boolean algebra, it is a Boolean algebra as well. The elements of $\mathcal{C}(A)$ are in fact equivalence classes of elements of $\mathcal{F}\left(A \times A^{\prime}\right)$. We will denote the equivalence class of a pair $(a, b) \in A \times A^{\prime}$ by $(a \mid b)$. Therefore, in $\mathcal{C}(A)$ we have basic conditionals of the form $(a \mid b)$ and compound conditionals, that is, those elements in $\mathcal{C}(A)$ that are algebraic terms definable in the language of Boolean algebras, modulo the identification induced by $\mathfrak{C}$. The operations on $\mathcal{C}(A)$ are denoted as follows, with the obvious interpretation

$$
\mathcal{C}(A)=\left(\mathcal{C}(A), \sqcap_{\mathfrak{C}}, \sqcup_{\mathfrak{C}}, \neg_{\mathfrak{C}}, \perp_{\mathfrak{C}}, \top_{\mathfrak{C}}\right) .
$$

In order to simplify our notation we will omit the subscript $\mathfrak{C}$ whenever this leads to no ambiguity.

Notice that, the above conditions $(\mathbf{t} 1)-(\mathbf{t} 5)$ used to define the quotient algebras $\mathcal{C}(A)$ automatically imply that, in any $\mathcal{C}(A)$, the following equations on conditionals will always hold: $(y \mid y)=\top,(x \mid y) \sqcap(z \mid y)=(x \wedge y \mid z), \neg(x \mid y)=$ $(\neg x \mid y),(x \wedge y \mid y)=(x \mid y)$, and $(x \mid y) \sqcap(y \mid z)=(x \mid z)$ whenever $x \leq y \leq z$.

Observe as well, that the basic conditionals of $\mathcal{C}\left(A, A^{\prime}\right)$ need not be closed under meets and joins, though in some particular cases they can be, for instance as a consequence of (t2) and (t 3$)$, ensuring that the conjunction of two basic conditionals with the same antecedent is a basic conditional, and the negation of a basic conditional, is basic as well. In any algebra $\mathcal{C}(A)$, as in any Boolean algebra, the induced order relation, also denoted $\leq$, is defined as $(x \mid y) \leq(z \mid$ $k)$ iff $(x \mid y) \sqcap(z \mid k)=(x \mid y)$.

Space constraints do not allow us to delve any further into BAC algebras. We refer the interested reader to [4] for further details, and limit ourselves to collect the properties required in this paper in the following proposition.

Proposition 1. In every $B A C$ algebra $\mathcal{C}(A)$, for every $x, z \in A$ and $y \in A^{\prime}$, the following properties hold:

(e1) $(y \mid y)=(\top \mid y)=\top$ and $(\neg y \mid y)=(\perp \mid y)=\perp$.

(e2) $(x \mid y) \sqcap(z \mid y)=(x \wedge z \mid y)$, and hence, if $x \wedge z=\perp$ then $(x \mid y) \sqcap(z \mid y)=\perp$.

(e3) if $z \in A^{\prime}$ and $x \leq y \leq z$ then $(x \mid z)=(x \mid y) \sqcap(y \mid z)$.

(e4) $(x \mid y) \sqcup(z \mid y)=(x \vee z \mid y)$ and $\neg(x \mid y)=(\neg x \mid y)$.

$(e 5)(x \wedge y \mid \top) \leq(x \mid y) \leq(\neg y \vee x \mid \top)$. 
(e6) $(x \mid \top) \sqcap(y \mid x) \leq(y \mid \top)$.

(e7) $(x \wedge y \mid \top) \leq(x \mid y)$ and $(\neg x \wedge y \mid \top) \leq \neg(x \mid y)$.

(e8) if $z \in A^{\prime}$ then $(x \mid y) \sqcap(x \mid z) \leq(x \mid y \vee z)$.

Interesting readings of some of the above properties are the following. $(e 1),(e 2)$ and (e4) force that for any $z \in A^{\prime}$, the set $A \mid z=\{(a \mid z) \mid a \in A\}$ is (the domain of) a Boolean subalgebra of $\mathcal{C}(A)$. On the other hand, $(e 5)$ shows that conditionals are stronger than material implications but weaker than conjunctions. Also, from a logical point of view, (e6) states a form of modus ponens with conditionals. $(e 7)$ states that, whenever $y$ unconditionally holds true, a conditional $(x \mid y)$ holds true if $x$ unconditionally holds true, while $(x \mid y)$ holds false otherwise. Finally, $(e 8)$ corresponds to the so-called OR property, typical in nonmonotonic systems, see Section 5.

\section{The atoms of a finite algebra of conditionals}

We now move on to the investigation of the atoms of a $\mathrm{BAC}$ algebra $\mathcal{C}(A)$ for a finite $A$. To this end it is worth to remember that an element $\alpha$ of a Boolean algebra $A$ is an atom of $A$ iff when $\alpha$ covers $\perp$, that is, $\perp<\alpha$ and if $\perp \leq \beta \leq \alpha$ then either $\beta=\perp$ or $\beta=\alpha$. Note that every algebra $\mathcal{C}(A)$ is finite whenever $A$ is. Indeed, if $A$ is finite, $\mathcal{F}\left(A \times A^{\prime}\right)$ is finite as well, since the variety of Boolean algebras is locally finite. Thus, $\mathcal{C}(A)$ is finite and hence atomic. In the following, we write $\operatorname{Atom}(B)$ to denote the set of atoms of any Boolean algebra $B$.

For the characterization theorem, we need some preliminary results. The following properties are immediate consequences of Proposition 1 (e3). In what follows $A$ will always denote a finite Boolean algebra such that $|\operatorname{Atom}(A)|=n$.

Lemma 1. Let $x, y, z \in A$. The following properties hold:

1. If $x \leq y \leq z$, then $(x \mid z) \leq(x \mid y)$; in particular $(x \mid \top) \leq(x \mid y)$.

2. If $x \wedge z=\perp$ and $\perp<x \leq y$, then $(x \mid \top) \sqcap(z \mid y)=\perp$.

Now we can prove the following interesting results.

Proposition 2. Let $i \leq n-1$ and define $\operatorname{Seq}_{i}(A)$ to be the set of sequences of length $i$ of pairwise distinct atoms of $A$. Then the set

$$
\begin{gathered}
\operatorname{Part}_{i}(\mathcal{C}(A))= \\
\left\{\left(\beta_{1} \mid \top\right) \sqcap\left(\beta_{2} \mid \neg \beta_{1}\right) \sqcap \ldots \sqcap\left(\beta_{i} \mid \neg \beta_{1} \wedge \ldots \wedge \neg \beta_{i-1}\right) \mid\left\langle\beta_{1}, \beta_{2}, \ldots, \beta_{i}\right\rangle \in \operatorname{Seq}_{i}(A)\right\}
\end{gathered}
$$

is a partition of $\mathcal{C}(A)$, that is, $\bigsqcup \operatorname{Part}_{i}(\mathcal{C}(A))=\top$ and for any distinct $C, D \in$ $\operatorname{Part}_{i}(\mathcal{C}(A)), C \sqcap D=\perp$.

Proof. (1) The case $i=1$ is easy as $\operatorname{Seq}_{1}(A)=\{\langle\alpha\rangle \mid \alpha \in \operatorname{Atom}(A)\}$, and it is clear that $\bigsqcup_{\alpha}(\alpha \mid T)=\left(\bigvee_{\alpha} \alpha \mid T\right)=T$.

(2) Suppose the claim is true for $i-1$, that is, $\bigsqcup \operatorname{Part}_{i-1}(\mathcal{C}(A))=\top$. Consider then a sequence $\bar{\beta}=\left\langle\beta_{1}, \ldots, \beta_{i-1}\right\rangle \in S e q_{i-1}$ and its corresponding compound conditional 
$H_{\bar{\beta}}=\left(\beta_{1} \mid \top\right) \sqcap \ldots \sqcap\left(\beta_{i-1} \mid \neg \beta_{1} \wedge \ldots \wedge \neg \beta_{i-2}\right)$. By hipothesis, we know that $\bigsqcup_{\bar{\beta} \in \text { Seq }_{i-1}} H_{\bar{\beta}}=\bigsqcup \operatorname{Part}_{i-1}(\mathcal{C}(A))=\top$.

Let $D(\bar{\beta})=\operatorname{Atom}(A) \backslash\left\{\beta_{1}, \ldots \beta_{i-1}\right\}$ be the set of $n-i+1$ atoms disjoint from $\left\{\beta_{1}, \ldots \beta_{i-1}\right\}$. Then it is clear that $\bigsqcup_{\beta \in D(\bar{\beta})}\left(\beta \mid \neg \beta_{1} \wedge \ldots \wedge \neg \beta_{i-1}\right)=\top$, and thus $H_{\bar{\beta}}=\bigsqcup_{\beta \in D(\bar{\beta})} H_{\bar{\beta}} \sqcap\left(\beta \mid \neg \beta_{1} \wedge \ldots \wedge \neg \beta_{i-1}\right)$.

Therefore, since we can do this for every sequence $\bar{\beta} \in S e q_{i-1}$, we finally get that

$\bigsqcup_{\bar{\beta} \in S_{e q} q_{i-1}} H_{\bar{\beta}}=\bigsqcup_{\bar{\beta} \in S_{e q_{i-1}}}\left(\bigsqcup_{\beta \in D(\bar{\beta})} H_{\bar{\beta}} \sqcap\left(\beta \mid \neg \beta_{1} \wedge \ldots \wedge \neg \beta_{i-1}\right)\right)=\bigsqcup_{\bar{\delta} \in S_{e q}} H_{\bar{\delta}}=\bigsqcup \operatorname{Part}_{i}(\mathcal{C}(A))$

Thus we have proved that $\bigsqcup \operatorname{Part}_{i}(\mathcal{C}(A))=\top$.

Let $A$ be a Boolean algebra with $n$ atoms. We denote by $\operatorname{Seq}(A)$ the set of sequences $\bar{\alpha}=\left\langle\alpha_{1}, \alpha_{2}, \ldots, \alpha_{n-1}\right\rangle$ of $n-1$ pairwise distinct atoms of $A$. Moreover, for every such a sequence $\bar{\alpha} \in \operatorname{Seq}(A)$, let us consider the compound conditional

$$
\omega_{\bar{\alpha}}=\left(\alpha_{1} \mid \top\right) \sqcap\left(\alpha_{2} \mid \neg \alpha_{1}\right) \sqcap \ldots \sqcap\left(\alpha_{n-1} \mid \neg \alpha_{1} \wedge \ldots \wedge \neg \alpha_{n-2}\right),
$$

or equivalently, $\omega_{\bar{\alpha}}=\left(\alpha_{1} \mid \top\right) \sqcap\left(\alpha_{2} \mid \alpha_{2} \vee \cdots \vee \alpha_{n}\right) \sqcap \ldots \sqcap\left(\alpha_{n-1} \mid \alpha_{n-1} \vee \alpha_{n}\right)$.

Theorem 1. The set of the atoms of $\mathcal{C}(A)$ is $\operatorname{Atom}(\mathcal{C}(A))=\left\{\omega_{\bar{\alpha}}: \bar{\alpha} \in \operatorname{Seq}(A)\right\}$. As a consequence, $\mid$ Atom $(\mathcal{C}(A)) \mid=n$ ! and $|\mathcal{C}(A)|=2^{n !}$.

Proof. We have to prove the following two conditions:

(i) For any $\omega_{\bar{\alpha}} \in \operatorname{Atom}(\mathcal{C}(A)), \omega_{\bar{\alpha}}>\perp$. First of all, observe that, looking at the way the set $\operatorname{Atom}(\mathcal{C}(A))$ is defined, if $\omega_{\bar{\alpha}}=\perp$ for some $\omega_{\bar{\alpha}} \in \operatorname{Atom}(\mathcal{C}(A))$ then, by a symmetry argument it would be the case that every $\omega_{\bar{\beta}} \in \operatorname{Atom}(\mathcal{C}(A))$ would also be $\perp$. Second, let us show that $\bigsqcup \operatorname{Atom}(\mathcal{C}(A))=\top$. Indeed, note that $\operatorname{Atom}(\mathcal{C}(A))=\operatorname{Part}_{n-1}(\mathcal{C}(A))$, and thus this directly follows from Proposition 2 when taking $i=n-1$. Therefore, we conclude that $\omega_{\bar{\alpha}}>\perp$ for every $\omega_{\bar{\alpha}} \in \operatorname{Atom}(\mathcal{C}(A))$.

(ii) For any $\omega_{\bar{\alpha}} \in \operatorname{Atom}(\mathcal{C}(A))$, there is no $D \in \mathcal{C}(A)$ such that $\perp<D<\omega_{\bar{\alpha}}$. It is enough to show that, for any element $(\gamma \mid b) \in \mathcal{C}(A) \backslash\{\perp\}$, with $\gamma \in \operatorname{Atom}(A)$, we have that either $\omega_{\bar{\alpha}} \sqcap(\gamma \mid b)=\perp$ or $\omega_{\bar{\alpha}} \sqcap(\gamma \mid b)=\omega_{\bar{\alpha}}$ itself. Since $\gamma \in \operatorname{Atom}(A)$, then $\gamma=\alpha_{i}$ for some $1 \leq i \leq n$. Then we have two cases: either $b=\alpha_{i} \vee \cdots \vee \alpha_{n}$, and in that case $\omega_{\bar{\alpha}} \sqcap(\gamma \mid b)=\omega_{\bar{\alpha}}$, or otherwise $b$ is of the form $b=\alpha_{i} \vee \alpha_{k} \vee a$, for some $k<i$. Then, in the latter case, we have $(\gamma \mid b) \sqcap\left(\alpha_{k} \mid \alpha_{k} \vee \ldots \vee \alpha_{n}\right)=\left(\alpha_{i} \mid \alpha_{i} \vee \alpha_{k} \vee a\right) \sqcap\left(\alpha_{k} \mid\right.$ $\left.\alpha_{k} \vee \ldots \vee \alpha_{n}\right) \leq\left(\alpha_{i} \mid \alpha_{i} \vee \alpha_{k}\right) \sqcap\left(\alpha_{k} \mid \alpha_{k} \vee \alpha_{i}\right)=\perp$, whence $(\gamma \mid b) \sqcap \omega_{\bar{\alpha}}=\perp$ as well.

Example 1. Let $A$ be the Boolean algebra of 3 atoms $\left\{\alpha_{1}, \alpha_{2}, \alpha_{3}\right\}$ and 8 elements. Theorem 1 tells us that the atoms of the algebra $\mathcal{C}(A)$ are as follows:

$$
\operatorname{Atom}(\mathcal{C}(A))=\left\{\left(\alpha_{i} \mid \top\right) \sqcap\left(\alpha_{j} \mid \neg \alpha_{i}\right): i, j=1,2,3 \text { and } i \neq j\right\} .
$$

Therefore, the algebra $\mathcal{C}(A)$, depicted in Figure 1 , has six atoms $\left\{x_{1}, \ldots, x_{6}\right\}$ and $2^{6}=64$ elements. In particular, we have that $x_{1}=\left(\alpha_{1} \mid \top\right) \sqcap\left(\alpha_{2} \mid \neg \alpha_{1}\right)$, $x_{2}=\left(\alpha_{1} \mid \top\right) \sqcap\left(\alpha_{3} \mid \neg \alpha_{1}\right), x_{3}=\left(\alpha_{2} \mid \top\right) \sqcap\left(\alpha_{1} \mid \neg \alpha_{2}\right), x_{4}=\left(\alpha_{2} \mid \top\right) \sqcap\left(\alpha_{3} \mid \neg \alpha_{2}\right)$, $x_{5}=\left(\alpha_{3} \mid \top\right) \sqcap\left(\alpha_{1} \mid \neg \alpha_{3}\right)$ and $x_{6}=\left(\alpha_{3} \mid \top\right) \sqcap\left(\alpha_{2} \mid \neg \alpha_{3}\right)$.

Let us consider the conditional $y=\left(\alpha_{1} \mid \neg \alpha_{3}\right)$. Obviously, $y=\bigsqcup\left\{x_{i}: x_{i} \leq y\right\}$ and, thanks to part (2) of Lemma 1, it is easy to see that, indeed, $y=x_{1} \sqcup x_{2} \sqcup x_{5}$. 


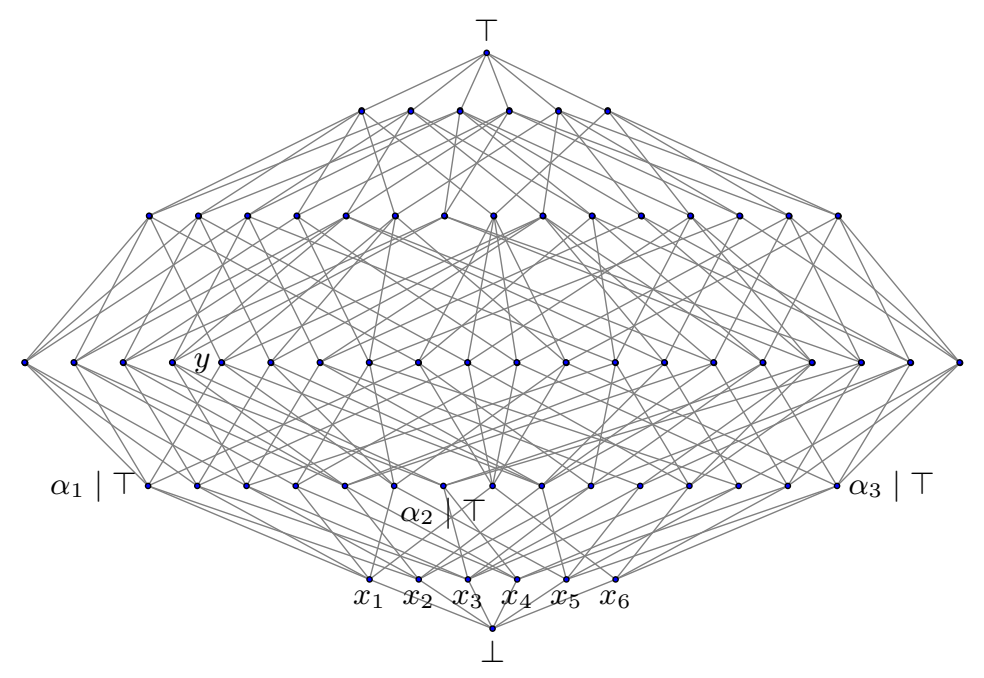

Fig. 1. The algebra of conditionals $\mathcal{C}(A)$, when $\mid$ Atom $(A) \mid=3$, the atoms of which are $x_{1}, x_{2}, x_{3}, x_{4}, x_{5}, x_{6}$. The element $y=\left(\alpha_{1} \mid \neg \alpha_{3}\right)$ is $x_{1} \sqcup x_{2} \sqcup x_{5}$.

As for a further explicative example, notice that $x_{1} \sqcup x_{2}=\left(\left(\alpha_{1} \mid \top\right) \sqcap\left(\alpha_{2} \mid\right.\right.$ $\left.\left.\neg \alpha_{1}\right)\right) \sqcup\left(\left(\alpha_{1} \mid \top\right) \sqcap\left(\alpha_{3} \mid \neg \alpha_{1}\right)\right)=\left(\alpha_{1} \mid \top\right) \sqcap\left(\alpha_{2} \vee \alpha_{3} \mid \neg \alpha_{1}\right)=\left(\alpha_{1} \mid \top\right) \sqcap\left(\neg \alpha_{1} \mid\right.$ $\left.\neg \alpha_{1}\right)=\left(\alpha_{1} \mid \top\right)$. Analogously $\left(\alpha_{2} \mid \top\right)=x_{3} \sqcup x_{4}$ and $\left(\alpha_{3} \mid \top\right)=x_{5} \sqcup x_{6}$.

We close this section by characterising the atoms which are below a given non-trivial basic conditional (i.e. different from $T$ ).

Lemma 2. Let $\bar{\alpha}=\left\langle\alpha_{1}, \alpha_{2}, \ldots, \alpha_{n-1}\right\rangle \in \operatorname{Seq}(A)$, and let $\omega_{\bar{\alpha}}$ be its corresponding atom, i.e. $\omega_{\bar{\alpha}}=\left(\alpha_{1} \mid \top\right) \sqcap\left(\alpha_{2} \mid \neg \alpha_{1}\right) \sqcap\left(\alpha_{3} \mid \neg \alpha_{1} \wedge \neg \alpha_{2}\right) \sqcap \ldots \sqcap\left(\alpha_{n-1} \mid\right.$ $\left.\neg \alpha_{1} \wedge \ldots \wedge \neg \alpha_{n-2}\right)$. Further let $\beta \in$ Atom $(A)$ and $y \in A$ such that $\beta<y$. Then,

$$
\omega_{\bar{\alpha}} \leq(\beta \mid y) \text { iff } \exists i \leq n-1 \text { such that } \alpha_{i}=\beta \text { and } \neg \alpha_{1} \wedge \ldots \wedge \neg \alpha_{i-1} \geq y .
$$

Proof. If $\alpha_{i}=\beta$ and $\neg \alpha_{1} \wedge \ldots \wedge \neg \alpha_{i-1} \geq y$, then clearly $\left(\alpha_{i} \mid \neg \alpha_{1} \wedge \ldots \wedge \neg \alpha_{i-1}\right) \leq(\beta \mid y)$ and hence $\omega_{\bar{\alpha}} \leq(\beta \mid y)$ as well. As for the other direction, we have two possibilities:

(i) There is $i \leq n-1$ such that $\alpha_{i}=\beta$. If $i=1$, then since $\top \geq y$, the condition is fulfilled. Then assume $i>1$ and $\omega_{\bar{\alpha}} \sqcap(\beta \mid y)=\omega_{\bar{\alpha}}$, we want to prove that $\neg \alpha_{1} \wedge \ldots \wedge$ $\neg \alpha_{i-1} \geq y$. Indeed, we have:

- If $\alpha_{1} \leq y$, we would have $\left(\alpha_{1} \mid \top\right) \sqcap(\beta \mid y) \leq\left(\alpha_{1} \mid y\right) \sqcap\left(\alpha_{i} \mid y\right)=\perp$, and hence $\omega_{\bar{\alpha}} \sqcap(\beta \mid y)=\perp$, contradiction. Therefore $\alpha_{1} \leq \neg y$.

- If $\alpha_{2} \leq y$, since $\alpha_{1} \leq \neg y$, we would have $\left(\alpha_{2} \mid \neg \alpha_{1}\right) \sqcap(\beta \mid y) \leq\left(\alpha_{2} \mid y\right) \sqcap(\beta \mid y)=\perp$, and hence $\omega_{\bar{\alpha}} \sqcap(\beta \mid y)=\perp$, contradiction. Therefore, $\alpha_{2} \leq \neg y$.

$-\ldots$

- If $\alpha_{i-1} \leq y$, since $\alpha_{1} \leq \neg y, \alpha_{2} \leq \neg y, \ldots, \alpha_{i-2} \leq \neg y$, we would have $\left(\alpha_{i-1} \mid\right.$ $\left.\neg \alpha_{1} \wedge \ldots \wedge \alpha_{i-2}\right) \sqcap(\beta \mid y) \leq\left(\alpha_{2} \mid y\right) \sqcap(\beta \mid y)=\perp$, , and hence $\omega_{\bar{\alpha}} \sqcap(\beta \mid y)=\perp$, contradiction. Therefore, $\alpha_{i-1} \leq \neg y$. 
As consequence, $\alpha_{1} \vee \ldots \vee \alpha_{i-1} \leq \neg y$ or, equivalently, $\neg \alpha_{1} \wedge \ldots \wedge \neg \alpha_{i-1} \geq y$.

(ii) $\beta=\alpha_{n}$, where $\alpha_{n}$ is the remaining atom not appearing $\bar{\alpha}$. In this case, one can show that $\omega_{\bar{\alpha}} \sqcap(\beta \mid y)=\perp$, and hence $\omega_{\bar{\alpha}} \not{Z}(\beta \mid y)$. Indeed, if $\beta=\alpha_{n}<y$, it means that $y \geq \alpha_{i} \vee \alpha_{n}$, with $i \leq n-1$. Then the atom $\omega_{\bar{\alpha}}$ contains the conjunct $\left(\alpha_{i} \mid \neg \alpha_{1} \wedge \ldots \neg \alpha_{i-1}\right)=\left(\alpha_{i} \mid \alpha_{i} \vee \ldots \vee \alpha_{n}\right)$, and we have $\omega_{\bar{\alpha}} \sqcap(\beta \mid y) \leq\left(\alpha_{i} \mid\right.$ $\left.\alpha_{i} \vee \ldots \vee \alpha_{n}\right) \sqcap(\beta \mid y) \leq\left(\alpha_{i} \mid \alpha_{i} \vee \alpha_{n}\right) \sqcap\left(\beta \mid \alpha_{i} \vee \alpha_{n}\right)=\perp$.

Proposition 3. Let $\bar{\alpha}=\left\langle\alpha_{1}, \alpha_{2}, \ldots, \alpha_{n-1}\right\rangle \in S e q(A)$, and let $\omega_{\bar{\alpha}}$ be its corresponding atom. Then, for any $x, y \in A \backslash\{\perp\}$ such that $x \ngtr y$.

$\omega_{\bar{\alpha}} \leq(x \mid y)$ iff $\exists i \leq n-1$ such that: (1) $\alpha_{i} \leq x \wedge y$, and (2) $\forall j<i, \neg \alpha_{j} \geq y$.

Proof. Since $(x \mid y) \neq \perp$, we know that $A t=\{\beta \in \operatorname{Atom}(A): \beta \leq x \wedge y\} \neq \emptyset$, and $(x \mid y)=\bigsqcup\{(\beta \mid y): \beta \in A t\}$. Now, $\omega_{\bar{\alpha}} \leq(x \mid y)$ iff there exists $\beta \in A t$ such that $\omega_{\bar{\alpha}} \leq(\beta \mid y)$. By Lemma 2, this holds iff there is $i \leq n-1$ such that $\alpha_{i}=\beta$ and for all $j<i, \neg \alpha_{j} \geq y$.

\section{Towards a logic for conditionals}

In this section we define first steps towards a logic to reason with conditionals whose semantics is in accordance with the notion of BAC algebras as described above. Let $\mathcal{L}$ be the classical propositional logic language, built from a finite set of propositional variables $p_{1}, p_{2}, \ldots p_{m}$. Based on $\mathcal{L}$, we define the language $\mathcal{C} \mathcal{L}$ of conditionals, in the style of e.g. [8], by the following stipulations:

- Atomic conditional formulas are expressions $(\varphi \mid \psi)$, where $\varphi, \psi \in \mathcal{L}$, and such that $\psi \forall \perp$. Atomic conditional formulas are in $\mathcal{C} \mathcal{L}$.

- Further, if $\Phi, \Psi \in \mathcal{C} \mathcal{L}$, then $\neg \Phi, \Phi \wedge \Psi, \Phi \vee \Psi \in \mathcal{C} \mathcal{L}{ }^{1}$

Definition 2. The Logic of Boolean conditionals (LBC for short) has the following axioms and rules, where $\vdash_{P L}$ denotes classical derivability:

$(P L)$ Axioms and rule of classical propositional logic for $\mathcal{C} \mathcal{L}$ formulas

(A1) $(\psi \mid \psi)$

(A2) $\neg(\varphi \mid \psi) \leftrightarrow(\neg \varphi \mid \psi)$

(A3) $(\varphi \mid \psi) \wedge(\delta \mid \psi) \leftrightarrow(\varphi \wedge \delta \mid \psi)$

(A4) $(\varphi \mid \psi) \leftrightarrow(\varphi \wedge \psi \mid \psi)$

(A5) $(\varphi \mid \psi) \leftrightarrow(\varphi \mid \chi) \wedge(\chi \mid \psi)$, if $\vdash_{P L} \varphi \rightarrow \chi$ and $\vdash_{P L} \chi \rightarrow \psi$

(R1) from $\vdash_{P L} \varphi \rightarrow \psi$ derive $(\varphi \mid \chi) \rightarrow(\psi \mid \chi)$

(R2) from $\vdash_{P L} \chi \leftrightarrow \psi$ derive $(\varphi \mid \chi) \leftrightarrow(\varphi \mid \psi)$

The notion of proof in $L B C, \vdash_{L B C}$, is defined as usual.

The above axiomatic system is clearly inspired on the key properties of BAC algebras, and indeed we can prove a tight relation with them. We shall write $\mathbb{L}$ to denote the Lindenbaum-Tarski algebra for the propositional language $\mathcal{L}$. For $\Phi, \Psi \in \mathcal{C} \mathcal{L}$, we write $\Phi \equiv \Psi$ if $\vdash_{L B C} \Phi \leftrightarrow \Psi$. (R1) and (R2) ensures that if $\vdash_{P L} \varphi \leftrightarrow \varphi^{\prime}$ and $\vdash_{P L} \psi \leftrightarrow \psi^{\prime}$, then $(\varphi \mid \psi) \equiv\left(\varphi^{\prime} \mid \psi^{\prime}\right)$. The following holds.

Proposition 4. The Lindenbaum-Tarski algebra for the language of conditionals $\mathcal{C} \mathcal{L}$, i.e. the quotient algebra $\mathcal{C} \mathcal{L} / \equiv$, is a $B A C$ algebra, namely $\mathcal{C L} / \equiv=\mathcal{C}(\mathbb{L})$.

\footnotetext{
${ }^{1}$ We use the same symbols for connectives in $\mathcal{L}$ and in $\mathcal{C} \mathcal{L}$ without danger of confusion.
} 


\section{Semantics and completeness}

The guiding idea in defining a semantics for $\mathcal{C L}$ is that the evaluations of $\mathcal{C} \mathcal{L}$ formulas should be in one-to-one correspondence with the atoms of the algebra $\mathcal{C L} / \equiv$. Recall from Section 3 that the atoms of $\mathcal{C}(A)$ are of the form

$$
\left(\alpha_{1} \mid \top\right) \sqcap\left(\alpha_{2} \mid \neg \alpha_{1}\right) \sqcap\left(\alpha_{3} \mid \neg \alpha_{1} \wedge \neg \alpha_{2}\right) \sqcap \ldots \sqcap\left(\alpha_{n-1} \mid \neg \alpha_{1} \wedge \ldots \wedge \neg \alpha_{n-2}\right),
$$

where $\alpha_{1}, \ldots \alpha_{n-1}$ are atoms of original algebra $A$, that is, the $\alpha_{i}$ 's must correspond to maximal elementary conjunctions of literals, or equivalently to evaluations of $\mathcal{L}$. In the following let $\Omega$ be the set of (classical) interpretations for the propositional language $\mathcal{L}$, i.e. $\Omega=\{w: \operatorname{Var} \rightarrow\{0,1\}\}$. Note that if there are $m$ propositional variables, then $|\Omega|=2^{m}$. Therefore, the idea is to define $\mathcal{C} \mathcal{L}$-evaluations as sequences $e=\left(w_{1}, \ldots, w_{2^{m}}\right)$, of pair-wise distinct $2^{m}$ interpretations $w_{1}, \ldots, w_{2^{m}} \in \Omega$, and to stipulate that such a $\mathcal{C L}$-evaluation $e$ makes true conditional $(\varphi \mid \psi)$ when the 'atomic' formula determined by $e$, $\left(\overline{w_{1}} \mid \top\right) \wedge\left(\overline{w_{2}} \mid \neg \overline{w_{1}}\right) \wedge \ldots \wedge\left(\overline{w_{n-1}} \mid \neg \overline{w_{1}} \wedge \ldots \wedge \neg \overline{w_{n-2}}\right)$, is 'below' $(\varphi \mid \psi)$, where $n=2^{m}$ and $\overline{w_{i}}$ denotes the maximal elementary conjunction of $\mathcal{L}$-literals that are true under $w_{i} \in \Omega$. Therefore, taking into account Proposition 3, we propose the following definition of $\mathcal{C} \mathcal{L}$-evaluations.

Definition 3. $A \mathcal{C} \mathcal{L}$-evaluation is a sequence $e=\left(w_{1}, w_{2}, \ldots, w_{n}\right)$ of $n$ pairwise distinct $w_{1}, \ldots, w_{n} \in \Omega$. The corresponding truth-evaluation of formulas of $\mathcal{C} \mathcal{L}$ is as follows:

- for atomic $\mathcal{C L}$-formulas: $e(\varphi \mid \psi)=1$ if $w_{i}=\varphi$ for the lowest index $i$ such that $w_{i}=\psi$, and $e(\varphi \mid \psi)=0$ otherwise.

- for compound $\mathcal{C} \mathcal{L}$-formulas: $e$ is extended using Boolean truth-functions.

The corresponding notion of consequence is as expected: for any set of $\mathcal{C L}$ formulas $\Gamma \cup\{\Phi\}, \Gamma \models_{L B C} \Phi$ if, for every $\mathcal{C} \mathcal{L}$-evaluation e such that $e(\Psi)=1$ for all $\Psi \in \Gamma$, then $e(\Phi)=1$.

In order to prove completeness for LBC, we need some preliminary results. For every valuation $h$ of the Lindenbaum algebra $\mathcal{C L} / \equiv$ into the 2-element Boolean algebra, i.e. for every Boolean homomorphism $h: \mathcal{C L} / \equiv \rightarrow\{0,1\}$, let $\omega^{h}$ be the unique atom in $\mathcal{C} \mathcal{L} / \equiv$ such that $h\left(\omega^{h}\right)=1$. Also, let $\overline{\alpha_{h}}=\left(\alpha_{1}, \ldots, \alpha_{n-1}\right)$ $\left(\right.$ for $\left.\alpha_{i} \in \operatorname{Atom}(\mathbb{L})\right)$ be such that $\omega^{h}=\omega_{\overline{\alpha_{h}}}$. Further, for every $\alpha_{i}$, let $w_{i}^{h}$ be the unique evaluation in $\Omega$ such that $w_{i}^{h}\left(\alpha_{i}\right)=1$. Then, we write $\Lambda(h)=$ $\left(w_{1}^{h}, \ldots, w_{n-1}^{h}\right)$.

Lemma 3. $\Lambda(h)$ is a $\mathcal{C} \mathcal{L}$-evaluation, and for each $\mathcal{C} \mathcal{L}$-formula $\Phi, h(\Phi)=1$ iff $\Lambda(h)(\Phi)=1$.

Proof. Let $h: \mathcal{C L} / \equiv \rightarrow\{0,1\}$ be a valuation as above. By construction, it is clear that $\Lambda(h)$ is a $\mathcal{C} \mathcal{L}$-evaluation. Now we prove, by induction on the structure of the formula $\Phi$, that $\Lambda(h)(\Phi)=1$ iff $h(\Phi)=1$. The interesting case is when $\Phi$ is an atomic conditional $(\varphi \mid \psi)$ such that $(\varphi \mid \psi) \not \equiv \top$. Then, $h(\Phi)=1$ iff $\omega^{h} \leq(\varphi \mid \psi)$ iff (by Proposition 3) there is $i \leq n-1$ such that $w_{i}^{h}(\varphi)=w_{i}^{h}(\psi)=1$ and $w_{l}^{h}(\psi)=0$ for all $l<i$, and thus, iff $\Lambda(h)=\left(w_{1}^{h}, \ldots, w_{n-1}^{h}\right)$ is such that a $\Lambda(h)(\Phi)=1$. 
Now the soundness and completeness of LBC easily follows from the above.

Theorem 2 (soundness and completeness). $L B C$ is sound and complete w.r.t. $\mathcal{C} \mathcal{L}$-evaluations, i.e. $\vdash_{L B C}=\models_{L B C}$.

Proof. Soundness is easy. As for completeness, assume that $\Gamma \forall_{L B C} \Phi$. Thus there exists a homomorphism $h: \mathcal{C L} / \equiv \rightarrow\{0,1\}$ such that $h(\gamma)=1$ for all $\gamma \in \Gamma$, and $h(\Phi)=0$. Thus, by Lemma $3, \Lambda(h)$ is a $\mathcal{C} \mathcal{L}$-evaluation such that $\Lambda(h)(\gamma)=1$ for every $\gamma \in \Gamma$ and $\Lambda(h)(\Phi)=0$, i.e. $\Gamma \nvdash_{L B C} \Phi$.

\section{Relation to non-monotonic reasoning models}

Conditionals possess an implicit non-monotonic behaviour. Given a conditional $(\varphi \mid \psi)$, it does not follow in general that we can freely strengthen its antecedent, i.e. in general, $(\varphi \mid \psi) \nvdash_{L B C}(\varphi \mid \psi \wedge \chi)$. For instance, $\varphi, \psi, \chi$ can be such that $\varphi \wedge \psi \not \models \perp$ while $\varphi \wedge \psi \wedge \chi \models \perp$. Actually, and not very surprisingly, the logic $\vdash_{L B C}$ satisfies the analogues of KLM-properties which characterize the wellknown system $\mathrm{P}$ of preferential entailment $[10,11]$.

Lemma $4 . \vdash_{L B C}$ satisfies the following properties:

Reflexivity: $\vdash_{L B C}(\varphi \mid \varphi)$

Left logical equivalence: if $\models_{P L} \varphi \leftrightarrow \psi$ then $(\chi \mid \varphi) \vdash_{L B C}(\chi \mid \psi)$

Right weakening: if $\models_{P L} \varphi \rightarrow \psi$ then $(\varphi \mid \chi) \vdash_{L B C}(\psi \mid \chi)$

Cut: $(\varphi \mid \psi) \wedge(\chi \mid \varphi \wedge \psi) \vdash_{L B C}(\chi \mid \psi)$

$O R:(\varphi \mid \psi) \wedge(\varphi \mid \chi) \vdash_{L B C}(\varphi \mid \psi \vee \chi)$

$A N D:(\varphi \mid \psi) \wedge(\delta \mid \psi) \vdash_{L B C}(\varphi \wedge \delta \mid \psi)$

Cautious Monotony: $(\varphi \mid \psi) \wedge(\chi \mid \psi) \vdash_{L B C}(\chi \mid \varphi \wedge \psi)$

Proof. Reflexivity, Left Logical Equivalence, Right Weakening and AND correspond to (A1), (R2), (R1), and (A3) of LBC, respectively. The other cases are proved as follows. Cut: by (A4), $(\chi \mid \varphi \wedge \psi) \wedge(\varphi \mid \psi)$ is equivalent to $(\chi \wedge \varphi \wedge \psi \mid \varphi \wedge \psi) \wedge(\varphi \wedge \psi \mid \psi)$, and by (A5), it is equivalent to $(\chi \wedge \varphi \wedge \psi \mid \psi)$, and by (R1) this clearly implies $(\chi \mid \psi)$.

Cautious Monotony: by (A3), $(\varphi \mid \psi) \wedge(\chi \mid \psi)$ is equivalent to $(\varphi \wedge \chi \mid \psi)$, which by (A 4) is in turn equivalent $(\varphi \wedge \chi \wedge \psi \mid \psi)$, and by (A5) implies $(\varphi \wedge \chi \wedge \psi \mid \varphi \wedge \psi$ ), which by (A3) it is equivalent to $(\chi \mid \varphi \wedge \psi)$.

$O R:(\varphi \mid \psi) \wedge(\varphi \mid \chi)$ is equivalent to $[(\varphi \mid \psi) \wedge(\varphi \mid \chi) \wedge(\psi \mid \psi \vee \chi)] \vee[(\varphi \mid \psi) \wedge(\varphi \mid$ $\chi) \wedge(\chi \mid \psi \vee \chi)]$, and this implies $[(\varphi \wedge \psi \mid \psi) \wedge(\psi \mid \psi \vee \chi)] \vee[(\varphi \wedge \chi \mid \chi) \wedge(\chi \mid \psi \vee \chi)]$, that is equivalent to $(\varphi \wedge \psi \mid \psi \vee \chi) \vee(\varphi \wedge \chi \mid \psi \vee \chi)$, which finally implies $(\varphi \mid \psi \vee \chi)$.

Now, let us fix a set of (atomic) conditional statements $K$, and let us define the consequence relation associated to $K: \varphi \sim_{K} \psi$ if $K \vdash_{L B C}(\psi \mid \varphi)$. Our last proposition is easily derived from the previous lemma.

Proposition 5. $\sim_{K}$ is a preferential consequence relation.

It can also be proved, but we omit this owing to space constraints, that Rational Monotonicity is also satisfied by LBC. Though this property is far more controversial than Cautious Monotonicity, it has been argued for in a number of circumstances. 


\section{Concluding remarks}

This paper deepens the investigation on Boolean algebras of conditional events that we began in [4]. Here, we have presented a full description of the atomic structure of these algebras and, based on this, we have defined a corresponding logic, LBC, to reason with conditionals. Moreover, we have shown tight connections of this logic with preferential nonmonotonic consequence relations.

Our previous work [4] was motivated by investigating the relationship of conditional probabilities on a Boolean algebra $A$ and simple probabilities on the algebras of conditional events $\mathcal{C}(A)$. Our conjecture is that for any conditional probability on $A$ there is a simple probability on $\mathcal{C}(A)$ agreeing on basic conditionals. Based on our new results, a close investigation on probabilities and other uncertainty measures on BAC algebras is the object of our future research.

Acknowledgments We are thankful to the anonymous reviewers. Godo acknowledges partial support by the Spanish FEDER/MINECO project TIN2015-71799-C2-1-P.

\section{References}

1. E.W. Adams. What is at stake in the controversy over conditionals. In Proceedings of WCII 2002, LNCS 3301, Springer-Verlag, 1-11, 2005.

2. P. Calabrese. An algebraic synthesis of the foundations of logic and probability. Information Sciences 42: 187-237, 1987.

3. D. Dubois, H. Prade. Measure-free conditioning, probability and non-monotonic reasoning. Proc. of IJCAI'89, Vol 2. pp. 1110-1114, 1989.

4. T. Flaminio, L. Godo and H. Hosni. On the Algebraic Structure of Conditional Events. Proc. ECSQARU'2015, S. Destercke et al. (Eds.), LNCS 9161, 106-116, 2015.

5. N. Friedman, J.Y. Halpern. Plausibility measures and default reasoning. Journal of the ACM, 48(4), 648-685, 2001.

6. A. Gilio and G. Sanfilippo. Conditional Random Quantities and Compounds of Conditionals. Studia Logica 102(4): 709-729, 2014.

7. I. R. Goodman, H. T. Nguyen and E. A. Walker. Conditional Inference and Logic for Intelligent Systems - A Theory of Measure-free Conditioning, North-Holland, 1991.

8. J.Y. Halpern. Defining Relative Likelihood in Partially Ordered Structures. Journal of Artificial Intelligence Research 7, 1-24, 1997.

9. G. Kern-Isberner. Conditionals in Nonmonotonic Reasoning and Belief Revision. Lecture Notes in Artificial Intelligence 2087, Springer, 2001.

10. D. Lehmann and M. Magidor. What does a conditional knowledge base entail?. Artificial Intelligence 55(1) 1-60, 1992.

11. D. Makinson. Bridges From Classical to Non-monotonic Logic. College Publications, London, 2005

12. D. Makinson. Conditional Probability in the Light of Qualitative Belief Change. In H. Hosni \& F. Montagna (Eds.), Probability, Uncertainty and Rationality, Edizioni della Normale, 2010.

13. P. Milne. Bruno de Finetti and the Logic of Conditional Events. British Journal for the Philosophy of Science 48, 2, 195-232, 1997.

14. H. T. Nguyen, E. A. Walker. A History and Introduction to the Algebra of Conditional Events and Probability Logic. IEEE Transactions on Systems, Man, and Cybernetics, Vol. 24, no. 12, pp. 1671-1675, December 1994.

15. G. Schay. An algebra of conditional events. Journal of Mathematical Analysis and Applications. 24 : 334-344, 1968. 